\title{
Supplementary Information for Photoluminescence Switching Effect in a Two-dimensional Atomic Crystal
}

Zheng Sun ${ }^{1,2^{*} \ddagger}$, Ke Xu ${ }^{3,4,5 \ddagger}$, Chang Liu ${ }^{1,6 \ddagger}$, Jonathan Beaumariage ${ }^{2}$, Jierui Liang ${ }^{2}$, Susan K Fullerton-Shirey ${ }^{3}$, Zhe-Yu Shi ${ }^{1}$, Jian $\mathrm{Wu}^{1,7,8}$, and David Snoke ${ }^{2}$

${ }^{1}$ State Key Laboratory of Precision Spectroscopy, East China Normal University, Shanghai, 200241, China

${ }^{2}$ Department of Physics and Astronomy, University of Pittsburgh, Pittsburgh, PA 15260, USA

${ }^{3}$ Department of Chemical and Petroleum Engineering, University of Pittsburgh, Pittsburgh, PA 15260, USA

${ }^{4}$ School of Physics and Astronomy, Rochester Institute of Technology, Rochester NY 14623, USA

${ }^{5}$ Microsystems Engineering, Rochester Institute of Technology, Rochester NY 14623, USA

${ }^{6}$ Institute for Advanced Study, Tsinghua University, Beijing 100084, China

${ }^{7}$ Collaborative Innovation Center of Extreme Optics, Shanxi University, Taiyuan, Shanxi 030006, China

${ }^{8}$ CAS Center for Excellence in Ultra-intense Laser Science, Shanghai 201800, China

(*Email: zsun@1ps.ecnu.edu.cn, ${ }^{\star}$ Contributed equally to this work) 


\section{Optical microscope image of CVD sample:}

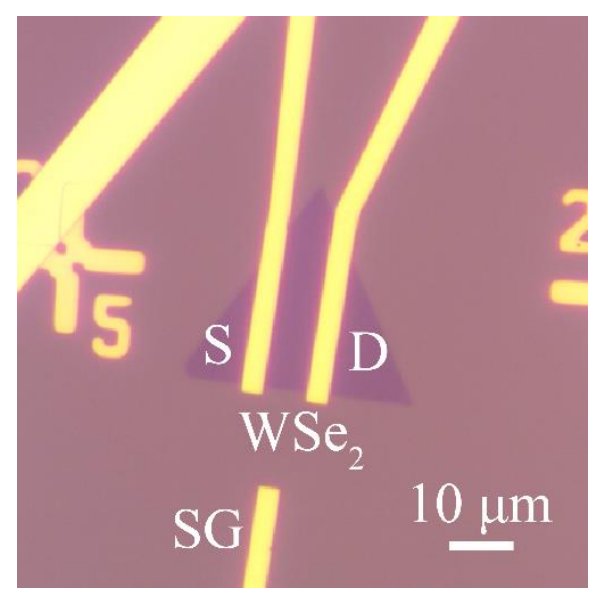

Fig. S1. Optical microscope image of CVD grown transistor with a side-gate. The tri-angular domain is the monolayer regime.

Transfer characteristics of CVD sample prior to electrolyte deposition.

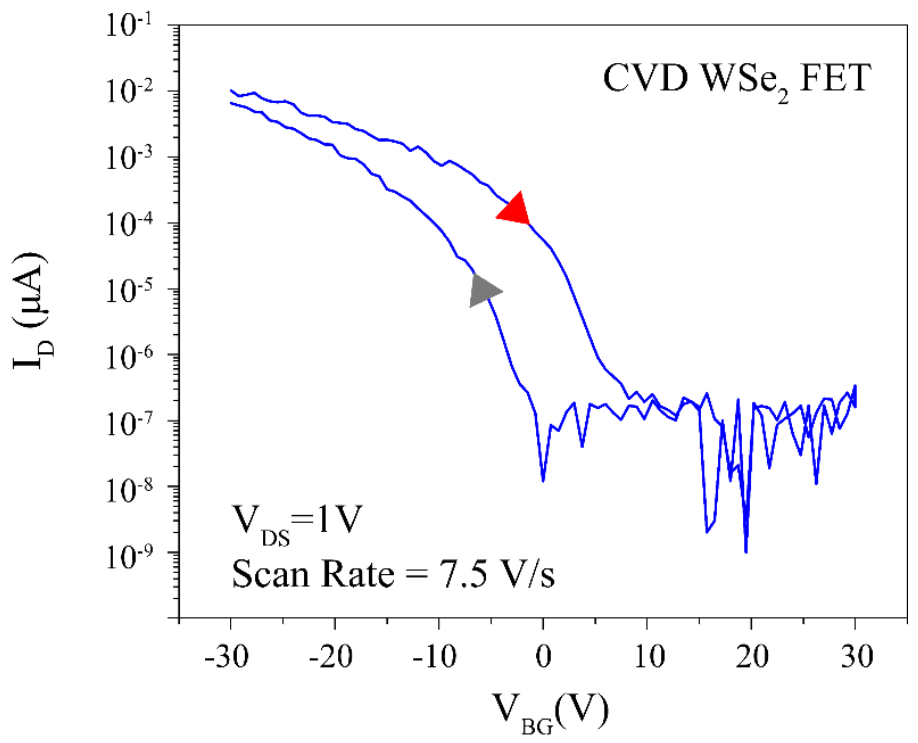

Fig. S2. Back-gate transfer characteristics of CVD WSe 2 FET prior to electrolyte deposition. Only a $p$-branch is observed within the measurement window, indicating p-type doping of the as-grown material. 


\section{PL intensity modulation using single vs dual gates on CVD sample}

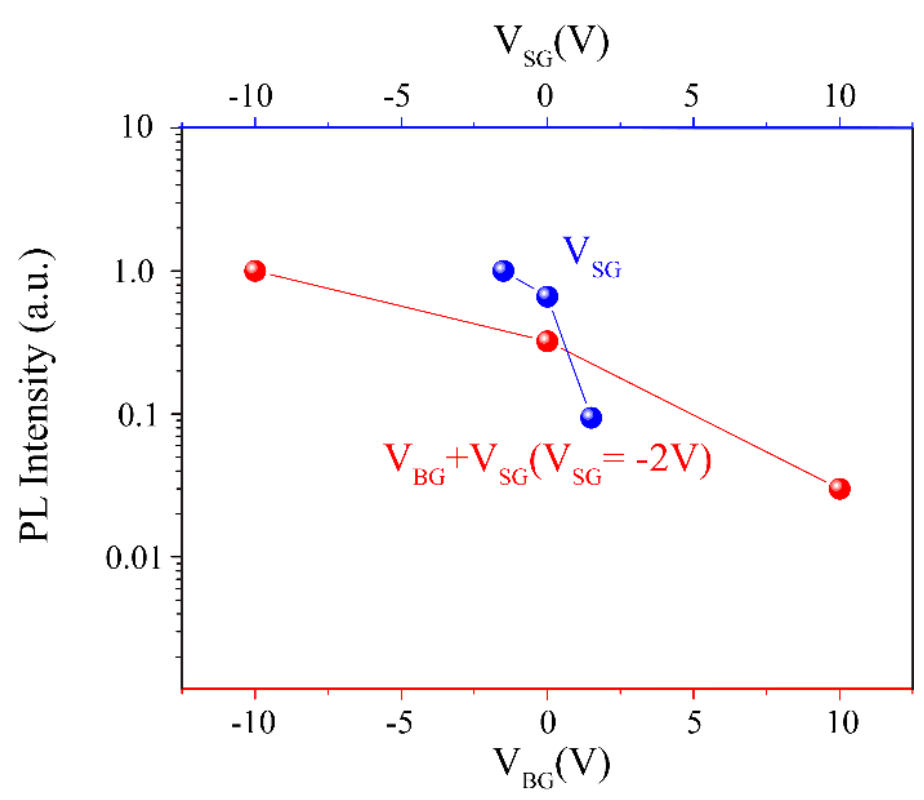

Fig. S3. PL intensity modulation with only side gate (blue) and dual gates (red). An on/off ratio of 12 is observed with only the side-gate, and the ratio is of 37 for the dual gate, in agreeing with our discussion of the mechanisms of PL intensity modulation.

Fig. S3 gives the results of a control experiment which was carried out to decouple the effect of the top and bottom electric field in the CVD-grown sample, by applying only the side-gate voltage and the dual gates. As expected, the change of the PL intensity can still be observed under the single side-gate conditions, but the on/off ratio is reduced to 12 . These results suggest that the PL switching effect is stronger when both gates are applied with vertical electric fields aligned in the same direction. 


\section{Optical switching for different polarity.}

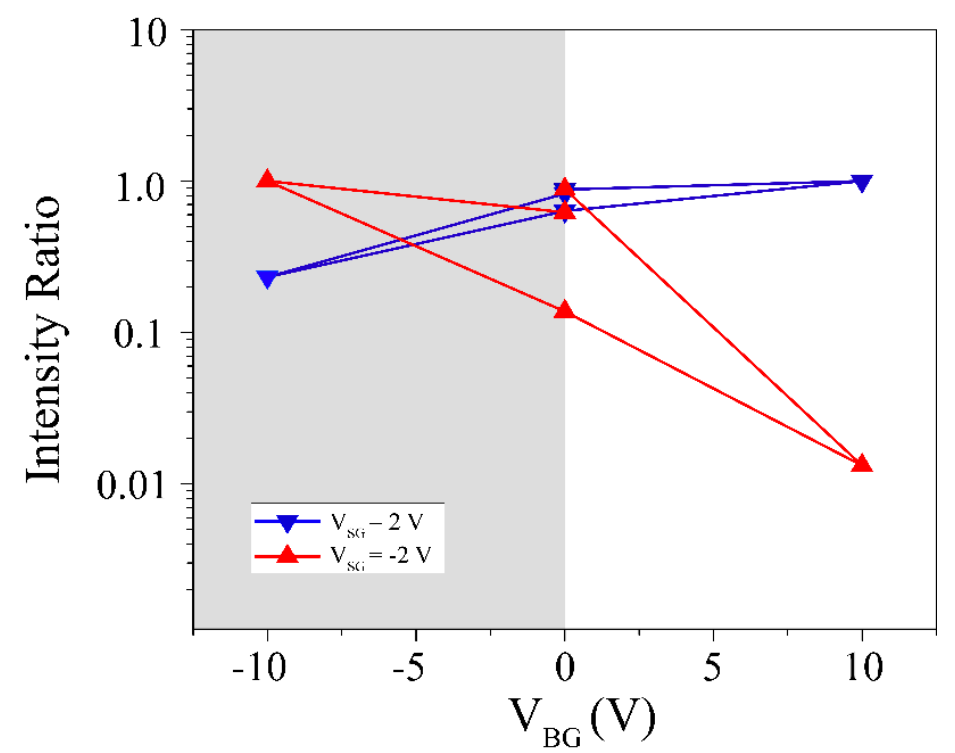

Fig. S4 PL intensity of the CVD WSe2-based transistors as the function of the back-gate voltage with initially set the side-gate voltage of $-2 \mathrm{~V}$ (in red) and $2 \mathrm{~V}$ (in blue). The on/off ratios were 37 and 7 respectively. All the data points are normalized by the maximum intensity.

As discussed in the manuscript, the device turns off when the EDL $\left(\mathrm{V}_{\mathrm{SG}}\right)$ induced electric field is in the same direction as $\mathrm{V}_{\mathrm{BG}}$ induced field. This is still true when side gate voltage is changed from $-2 \mathrm{~V}$ to $+2 \mathrm{~V}$, and the device turns off when $\mathrm{V}_{\mathrm{BG}}$ is negative and have the same direction of $\mathrm{V}_{\mathrm{SG}}$ field. However, the on/off ratio when $\mathrm{V}_{\mathrm{SG}}$ is $+2 \mathrm{~V}$ is about 7 , lower than the ratio of 37 when $V_{S G}$ is $-2 \mathrm{~V}$. We don't think it's majorly the impact of ionic species because our previous experimental results using Hall Effect measurement and transfer measurement, ${ }^{1,2}$ and simulation results using finite element modeling ${ }^{3}$ both suggest that the cationic and anionic EDL have very similar density. More devices and experiments would be needed to have a conclusive understanding. One possible explanation for this difference in PL on/off ratio might be the intrinsic p-type doping of the CVD grown WSe2 sample, which can be seen from the transfer measurement in Fig. S2. At the positive voltage the current, therefore charge carrier density, is lower than at the negative voltage, which may indicate we need different voltages to align exciton dipoles to turn on/off PL. It is an interesting effect that we can investigate in a future study. 


\section{Nonlinear optical susceptibility and the blue shift of PL energy}

The screened Coulomb interaction in a monolayer material is given by the Keldysh potential ${ }^{4-}$ ,

$$
V_{K}(r)=-\frac{e^{2}}{8 \epsilon_{0} r_{0}}\left[H_{0}\left(\frac{\kappa r}{r_{0}}\right)-Y_{0}\left(\frac{\kappa r}{r_{0}}\right)\right],
$$

where the screening length $r_{0}=\left(\epsilon_{\|}-1\right) d / 2$ depends on the layer separation $\mathrm{d}$ and in-plane dielectric constant $\epsilon_{\|}$, and the average dielectric constant $\kappa=\left(\epsilon_{\text {top }}+\epsilon_{\text {bottom }}\right) / 2$ (we take $\kappa=$ 4.5 as the same value taken in the literature ${ }^{4-9}$ ).

Under a strong vertical electric field, the dielectric constant can be expressed as $\epsilon_{\|}=$ $\epsilon_{0}\left(1+\chi^{(1)}+\chi^{(2)} E_{\perp}+\chi^{(3)} E_{\perp}^{2}+\cdots\right)$, where $\chi^{(\mathrm{n})}, n=1,2,3$ stand for the $\mathrm{nth}$ order (off-diagonal) optical susceptibilities. In a uniform medium, the second order nonlinear susceptibility $\chi^{(2)}$ is ignored ${ }^{10}$ and we only consider the third order nonlinear susceptibility $\chi^{(3)}$. The screening length $r_{0}$ then has a quadratic dependence on the vertical electric field because of $\chi^{(3)}$, which causes the change of exciton binding energy and eventually leads to the blue shift of the PL signal.

We numerically calculated the binding energy for different screening length $r_{0}$ according via the two-body Schrödinger equation,

$$
\frac{\hbar^{2} \nabla^{2}}{2 m_{r}} \phi(\mathbf{r})+V_{K}(r)=E \phi(\mathbf{r})
$$

with $m_{r}=0.2 m_{e}$ being the reduced effective mass of electron and hole. The binding energy is then fitted with the experimental measured PL blueshift and gives the off-diagonal optical susceptibility $\chi^{(3)}=3.50 \times 10^{-19} \mathrm{~m}^{2} / V^{2}$. 


\section{Capacitive coupling effect from electrolyte/oxide interface}
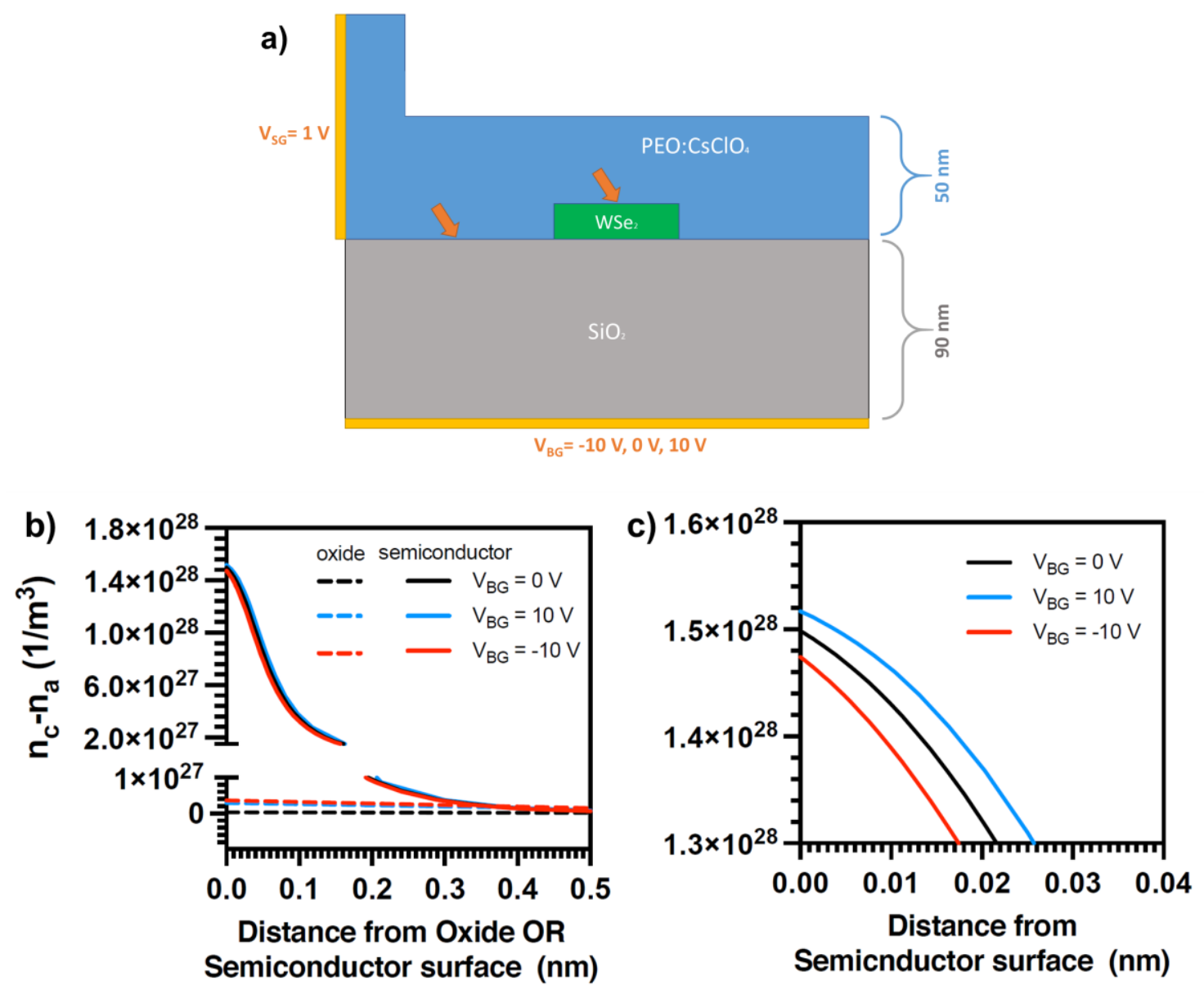

Fig. S5. a) Side gate device geometry setup in COMSOL. The arrows indicate locations where ion density are plotted in b) and c). b) Ion density as a function of distance from both the $\mathrm{WSe}_{2}$ (solid lines) and oxide surfaces (dashed lines) into the electrolyte at $\mathrm{V}_{\mathrm{BG}}=0,-10,+10 \mathrm{~V}$. c) Zoomed in view of EDL ion density at the electrolyte/ $\mathrm{WSe}_{2}$ interface.

Because EDL will form at both the electrolyte- $\mathrm{SiO}_{2}$ interface and the electrolyte- $\mathrm{WSe}_{2}$ channel interface, there is some capacitive coupling effect. However, to quantify whether or not this coupling is significant, we ran additional finite element modeling using COMSOL Multiphysics. We set the side gate equal to $1 \mathrm{~V}$ and the backgate to $-10,0$ or $+10 \mathrm{~V}$. A schematic of the sidegated device geometry and ion density as a function of distance from both the $\mathrm{WSe}_{2}$ and oxide surfaces into the electrolyte are provided in Fig. S5. The ion density in the EDL at the surface of the semiconductor is two orders of magnitude larger than that at the electrolyte/oxide interface. With the $\mathrm{V}_{\mathrm{BG}}$ set to $+10 \mathrm{~V}$ and the $\mathrm{V}_{\mathrm{SG}}$ set to $+1 \mathrm{~V}$ (i.e., the fields reinforce each other and would 
give the highest possible capacitive coupling), the EDL concentration on top of the $\mathrm{WSe}_{2}$ is $1.3 \%$ larger than when the $\mathrm{V}_{\mathrm{BG}}$ is set to zero. A $1.3 \%$ modulation on the $\mathrm{WSe}_{2}$ with the backgate shows that the $\mathrm{V}_{\mathrm{BG}}$ and the channel EDL are mostly independent of each other, and that capacitive coupling from the nearby oxide doesn't play a significant role.

\section{Reference:}

(1) Li, H. M.; Xu, K.; Bourdon, B.; Lu, H.; Lin, Y. C.; Robinson, J. A.; Seabaugh, A. C.; Fullerton-Shirey, S. K. Electric Double Layer Dynamics in Poly(ethylene oxide) LiClO4 on Graphene Transistors. J. Phys. Chem. C 2017, 121 (31), 16996-17004. https://doi.org/10.1021/acs.jpcc.7b04788.

(2) Xu, K.; Liang, J.; Woeppel, A.; Bostian, M. E.; Ding, H.; Chao, Z.; McKone, J. R.; Beckman, E. J.; Fullerton-Shirey, S. K. Electric Double-Layer Gating of TwoDimensional Field-Effect Transistors Using a Single-Ion Conductor. ACS Appl. Mater. Interfaces 2019, 11 (39), 35879-35887. https://doi.org/10.1021/acsami.9b11526.

(3) Woeppel, A.; Xu, K.; Kozhakhmetov, A.; Awate, S.; Robinson, J. A.; Fullerton-Shirey, S. K. Single- versus Dual-Ion Conductors for Electric Double Layer Gating: Finite Element Modeling and Hall-Effect Measurements. ACS Appl. Mater. Interfaces 2020, 12 (36), 40850-40858. https://doi.org/10.1021/acsami.0c08653.

(4) Stier, A. V.; Wilson, N. P.; Velizhanin, K. A.; Kono, J.; Xu, X.; Crooker, S. A. Magnetooptics of Exciton Rydberg States in a Monolayer Semiconductor. Phys. Rev. Lett. 2018, 120 (5), 1-6. https://doi.org/10.1103/PhysRevLett.120.057405.

(5) Keldysh, L. V. Coulomb Interaction in Thin Semiconductor and Semimetal Films. JETP Letters 1979, pp 658-661.

(6) Cudazzo, P.; Tokatly, I. V.; Rubio, A. Dielectric Screening in Two-Dimensional Insulators: Implications for Excitonic and Impurity States in Graphane. Phys. Rev. B Condens. Matter Mater. Phys. 2011, 84 (8), 1-7. https://doi.org/10.1103/PhysRevB.84.085406.

(7) Berkelbach, T. C.; Hybertsen, M. S.; Reichman, D. R. Theory of Neutral and Charged Excitons in Monolayer Transition Metal Dichalcogenides. Phys. Rev. B - Condens. Matter 
Mater. Phys. 2013, 88 (4), 1-6. https://doi.org/10.1103/PhysRevB.88.045318.

(8) Wu, F.; Qu, F.; Macdonald, A. H. Exciton Band Structure of Monolayer MoS2. Phys. Rev. B - Condens. Matter Mater. Phys. 2015, 91 (7), 1-8. https://doi.org/10.1103/PhysRevB.91.075310.

(9) Kylänpää, I.; Komsa, H. P. Binding Energies of Exciton Complexes in Transition Metal Dichalcogenide Monolayers and Effect of Dielectric Environment. Phys. Rev. B Condens. Matter Mater. Phys. 2015, 92 (20), 1-6. https://doi.org/10.1103/PhysRevB.92.205418.

(10) Boyd, R. W. Nolinear Optics; Elsevier: Amsterdam, 1967. 